\title{
Evidence of early multi-strange hadron freeze-out in high energy nuclear collisions
}

\author{
H. van Hecke ${ }^{a}$, H. Sorge ${ }^{b}$, and N. Xu ${ }^{c}$ \\ (a) MS H846, LANL, Los Alamos, NM 87545, USA \\ (b) Department of Physics, SUNY at Stony Brook, NY 11794, USA \\ (c) Nuclear Science Division, LBNL, Berkeley, CA 94720, USA
}

(September 26, 2018)

\begin{abstract}
Recently reported transverse momentum distributions of strange hadrons produced in $\mathrm{Pb}(158 \mathrm{AGeV})$ on $\mathrm{Pb}$ collisions and corresponding results from the relativistic quantum molecular dynamics (RQMD) approach are examined. We argue that the experimental observations favor a scenario in which multi-strange hadrons are formed and decouple from the system rather early at large energy densities (around 1 $\mathrm{GeV} / \mathrm{fm}^{3}$ ). The systematics of the strange and non-strange particle spectra indicate that the observed transverse flow develops mainly in the late hadronic stages of these reactions.
\end{abstract}

The purpose of the current and forthcoming heavy ion programs at the high-energy laboratories CERN (Switzerland) and Brookhaven National Laboratory (USA) is to probe strongly interacting matter under extreme conditions, i.e. at high densities and temperatures. The central subject of these studies is the transition from the quark-gluon plasma to hadronic matter. In the early phases of ultrarelativistic heavy ion collisions, when a hot, dense region is formed in the center of the reaction, there is copious production of up, down, and strange quarks. Transverse expansion is driven by the numerous scatterings among the incoming and produced particles. As the medium expands and cools, the quarks combine to form the hadrons that are eventually observed.

In this Letter we are going to address the question whether data from heavy ion experiments allow one to make statements about the existence of strange hadrons in a medium of high energy density $\epsilon>1 \mathrm{GeV} / \mathrm{fm}^{3}$. So far, experimental information about survival or "melting" of quark bound states was restricted to charmonium states only 11. On the other side, the strange quark mass is intermediate between charm and the very light flavors (up and down). Strangeness should in fact not be considered as an "external probe" of the hot medium like charm, as it may influence fundamental characteristics of the QCD phase transition itself, e.g. its order [2]. Furthermore, we argue in this Letter that the strange and non-strange hadron spectra encode information on the timing of flows and pressures. It has been recognized for a long time [3] that hadron momentum spectra are a valuable source of information on the collective flow developing in ultrarelativistic heavy ion collisions. The flows may be related to bulk and transport properties in the ultra-dense matter like transient pressure and viscosities. Presence of hydrodynamic behavior is expected at least for truly large colliding system such as $\mathrm{Pb}+\mathrm{Pb}$. Concerning the search for the quark-gluon plasma one would like to identify the regions in energy density at which the Equation of State (EOS) softens, presumably due to the phase transition or a cross-over between hadronic and quark matter. Another topic of interest is to see the EOS becoming hard again at yet higher densities. Asymptotically, the EOS approaches the Stefan-Boltzmann limit $p=\epsilon / 3$, where $p$ is the pressure, according to recent lattice calculations [4. Note, however, that the regime of perturbative quarks and gluons seems to be reached only at grand unification scales [5]. In the context of ultrarelativistic collisions the EOS dependence on energy and baryon density translates into a pressure dependence on time, because expansion dilutes the matter continuously. Here hadrons with varying strangeness content play an important role. With vastly differing reaction rates in the medium, they decouple at different times from the evolving system. Pictorially speaking, we may employ these spectra to get a sequence of snapshots of the transverse flow present at each of the species-dependent decoupling times.

On the experimental side, the predicted presence of strong radial transverse flow in the $\mathrm{Pb}(158 \mathrm{AGeV})$ on $\mathrm{Pb}$ collisions ( 0.4 to $0.6 \mathrm{c}$ ) [6] has been deduced from the systematics of non-strange particle spectra already some time ago [7,8]. The long awaited spectra of multiple strange hadrons $\Phi, \Xi$, and $\Omega$, measured at mid-rapidity, were reported during the Quark Matter '97 conference [9,10]. It came somewhat as a surprise that the reported slopes of these multi-strange hadrons ( $\Xi$ 's and $\Omega$ 's and possibly $\Phi$ 's) are much softer than expected from the trends in the previously observed mass dependence of the slope parameters [8].

In this Letter we will argue that these deviations find their natural explanation in a reaction scenario in which the multi-strange hadrons freeze out early, before sizable transverse flow has been developed. The corollary statement is that the transverse flow is a phenomenon emerging only later in the $\mathrm{Pb}$ on $\mathrm{Pb}$ reactions. In order to go beyond a qualitative interpretation we utilize a transport theoretical approach - relativistic quantum molecular dynamics (RQMD) - whose predictions agree well with the observations.

Several scenarios for the unknown earlier stages in ultrarelativistic nucleus-nucleus are imaginable and have been put forward. We will try to use the most recent information on the spectra to eliminate some of them. Let 
us list some of the possible choices:

- Is the expanding matter made of weakly interacting quarks and gluons, or of bound states (hadrons)?

- Does the flow appear already at an early stage of the collision [11] or rather later [12]?

- Does freeze-out occur instantaneously [13] or sequentially [14,15]?

We do not believe that the existence of strong transverse flow in the $\mathrm{Pb}(158 \mathrm{AGeV})$ on $\mathrm{Pb}$ reactions belongs to the unresolved questions. It is well-known that the difference between flow - a space-momentum correlation - and excitations purely in momentum space (temperature or random kicks [16]) shows up in observables which are sensitive to the phase-space densities of the finally emitted hadrons (like nucleon cluster formation 6 and HBT [17).

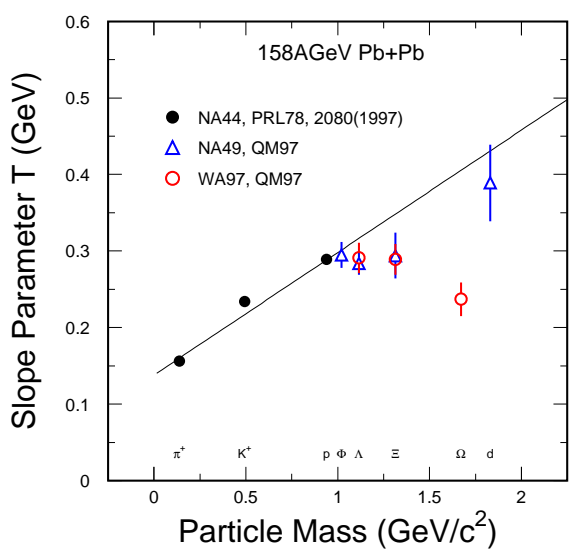

FIG. 1. Measured slope parameters as a function of particle mass. Preliminary results of the slope parameters of strange particles are shown with open symbols. The line represents the parameterization mentioned in the text.

Let us turn now to the recent data and interpret them in the light of these ideas. A compilation of the experimental data is shown in Fig. 1. The non-strange data may be parameterized by $T=T_{f o}+m\left\langle\beta_{t}\right\rangle^{2}$, where $T$ the slope parameter at mid-rapidity [18 and $m$ the particle mass. The parameter values $T_{f o} \approx 145 \mathrm{MeV},\left\langle\beta_{t}\right\rangle=0.4 c$. Recent reports show that this trend is not only obeyed by $\pi^{+/-}, p, \bar{p}$ and deuterons but also by hadrons containing one single strange quark [8,10]. However, note the strong deviation of the multiple strange baryon $\left(\Xi^{-}, \Omega\right)$ slopes from the general trends. How much the $\Phi$ deviates from the general systematics is not yet settled [19].

We may draw three conclusions from the compilation of slope parameters. The frequently employed picture one-fluid flow until break-up of the matter at a common freeze-out state - is untenable in view of these data. Such a picture results in a unique dependence of slope parameters on the particle mass, in clear contrast to the observations. Note that feed-down corrections which could spoil the universal dependence are of relevance mostly for pions but not the "heavy" hadron sector (with masses close to and above $1 \mathrm{GeV}$ ). Furthermore, the striking difference between multi-strange hadron and the other hadron slopes points to dynamics in the hadronic stages that causes the non-universal pattern. It is well-known from the early ideas of "strangeness as a QGP signal" 20 that strange flavor impacts reactions in quark matter rather differently than in a hadron gas. For instance, perturbative QGP interactions at relevant temperatures are only mildly affected by the light quark mass variations (5$160 \mathrm{MeV}$ ). On the other side, transport properties of the heavy $\Omega$ baryons with its mass of $1672 \mathrm{MeV}$ are expected to differ completely from the almost massless pions (140 $\mathrm{MeV}$ [21]. Finally, we note that the $\Xi$ slope parameters in $\mathrm{Pb}$ on $\mathrm{Pb}$ collisions are comparable to those in $\mathrm{p}$ on $\mathrm{W}$ and $\mathrm{S}$ on $\mathrm{W}$ reactions 22]. This fact provides strong constraints on any reaction dynamics. While much larger energy densities are surely produced in the heavy-ion collisions it does not seem to affect the transverse motion of particles like the $\Xi$. Scenarios in which the precursor matter experiences sizable flow before freeze-out of the multi-strange hadrons seem therefore to be ruled out by the data 23]. The conclusion that there can be only a tiny collective component in the transverse momentum spectra of the multi-strange hadrons is corroborated by an analysis of the chemical composition. The slope parameter $237 \pm 24 \mathrm{MeV}$ of the $\Omega$ particle is consistent with the "temperature" value extracted from the particle ratios 24.

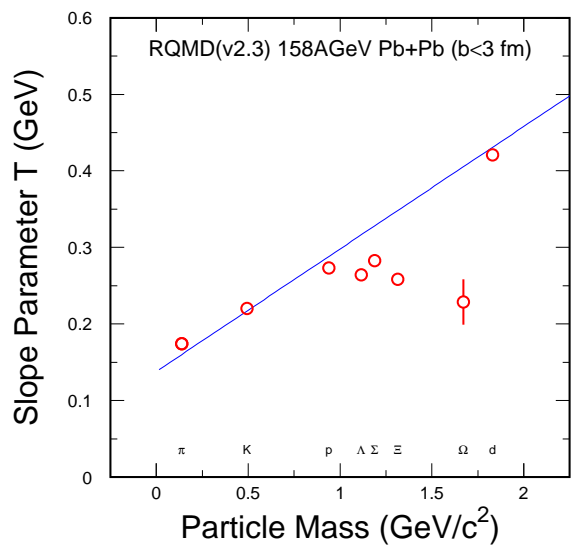

FIG. 2. The RQMD 2.3 model prediction of the slope parameters.

We now turn to the RQMD calculations in order to go beyond qualitative statements. The RQMD model 25] provides a microscopic description of heavy ion collisions which has been highly successful in predicting most of the observed features over a wide range of conditions. We 
have generated 1600 events for $\mathrm{Pb}(158 \mathrm{AGeV})$ on $\mathrm{Pb}$ employing RQMD (version 2.3) and analyzed the final spectra in the same fashion as has been done with the measurements. Before we analyze the aspects of the RQMD evolution dynamics which are pertinent for our discussion we present the results for the slope parameters of the various species in Fig. 2. Very good agreement is found between RQMD predictions and preliminary data.

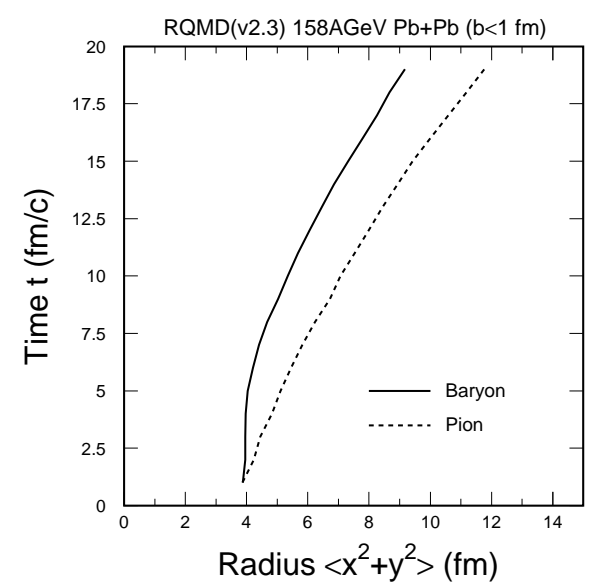

FIG. 3. Time evolution of the transverse source size for mid-rapidity baryons and pions.

The transverse expansion dynamics generated by RQMD may be schematically decomposed into two stages. The pre-equilibrium stage is determined by the initial excitation and fragmentation of color strings and ropes followed by kinetic equilibration in an ultradense hadron gas. During this stage the effective transverse pressure is ultrasoft which is a result of the combined effects from initial collision geometry, string dynamics which keeps a memory of the original beam axis, finite mean free paths and creation of "resonance matter" 12 . According to the RQMD calculations this stage lasts for about $5 \mathrm{fm} / \mathrm{c}$ in central $\mathrm{Pb}$ on $\mathrm{Pb}$ reactions. The state when local kinetic equilibrium is finally achieved is soon followed by a break-down of equilibrium due to the diluteness of the hadron gas and the finite size of the system. It has also been shown that the RQMD evolution of the multi-component hadronic fluid is characterized by non-ideal effects, even in the dense regime 15. The pions accelerate quite easily, their motion more or less governed by their own EOS. In contrast, the rarer heavy particles cannot keep up with the pion "fluid" and are left behind. The developing flow of matter according to the RQMD calculations is illustrated by plotting the mean transverse distance of hadrons from the center of the collision region (see Fig. 3). We see from Fig. 3 that the baryon matter does not expand at all during the first $5 \mathrm{fm} / \mathrm{c}$. Only after the soft stage has elapsed the baryons develop collective flow which is reflected in the increase of their spatial distribution. Essentially all of the transverse baryon flow is created during the late stage. Owing to the different transverse sizes of the collision region this stage lasts much longer in $\mathrm{Pb}$ on $\mathrm{Pb}$ than in reactions with smaller projectiles and leads to the marked difference between momentum spectra for $\mathrm{Pb}+\mathrm{Pb}$ as compared to $\mathrm{p}+\mathrm{A}$ and $\mathrm{S}+\mathrm{A}$ reactions.

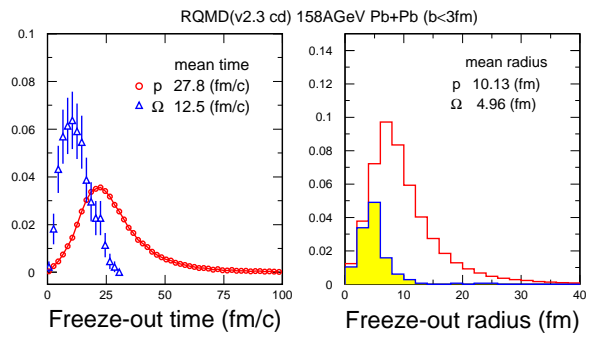

FIG. 4. The time and transverse radius distributions of midrapidity $\Omega$ 's and nucleons at freeze-out in central $\mathrm{Pb}+\mathrm{Pb}$ collisions at $158 \mathrm{AGeV}$ from RQMD.

Why are the multiple strange particles not dragged with the heavy particle flow? Their interactions in the expansion stage are dominated by resonance formation. A good measure of the reaction rates are therefore the decay widths of the baryon resonances to which the baryons couple. We see from the particle data group tables that the decay widths of resonances is a strong function of their flavor content [26]. Approximately, the trend is 0.45:0.62:0.85:1 for $\Omega^{*}: \Xi^{*}: Y^{*}: N^{*}$. We expect from these numbers that the $\Xi$ collision rates will be suppressed by on the order of 30 to 40 percent compared to $\Lambda$ 's and nucleons. Furthermore, the $\Omega$ is basically not involved in forming these resonances. A reason is that the $\pi$ - $\Omega$ system does not match any of their flavor quantum numbers. Furthermore, $\Omega+\eta$ and $\Omega+K$ collisions are suppressed due to the comparably rarer meson partners. The physics of resonance formation is built into the RQMD approach (for details see 25]). RQMD predicts considerably earlier freeze-out of $\Omega$ baryons (and $\Xi$ 's) than nucleons (and $\Lambda$ 's). The time and spatial distributions of nucleons and $\Omega$ 's are displayed in Fig. 4. Most of the $\Omega$ 's freeze-out between 2 and $8 \mathrm{fm} / \mathrm{c}$ while the nucleon freeze-out distribution is centered around $20 \mathrm{fm} / \mathrm{c}$. Note that the freezeout time spectra are characterized by sizable differences between median and averages. Very soft collisions between particles of almost equal velocities lead to the long tails in the distributions but are of no importance for the reaction kinetics.

The corresponding transverse distance distribution which is also shown in Fig. 4 reveals that the $\Omega$ source at freeze-out is very similar to the initial source. In contrast, nucleons are transported by the collective flow into outer shells. We can analyze the RQMD results concerning the local energy densities at which the multi-strange hadrons decouple from the system. Typically, these local energy densities cluster around $1 \mathrm{GeV} / \mathrm{fm}^{3}$. Clearly, this sets a lower limit for the densities at which these particles may form as quark bound states. 
We expect that the RQMD evolution in the late dilute hadron gas stage is calculated rather accurately - under the assumed given initial conditions. For this stage (and only here) our inability to calculate nucleus-nucleus reactions based on quantum chromodynamics (QCD) can be compensated. Utilizing the empirical information about the interactions between hadrons, kinetic equations can be set up and solved like in RQMD and other approaches [25], 227 29]. Theoretical justification for semi-classical transport comes essentially from the particle-like behavior with the DeBroglie wavelengths being typically much smaller than the mean free paths. Of course, the final hadron momentum spectra are a product of the timeintegrated dynamics starting with the initial interpenetration of the two nuclei. The agreement between RQMD and experimental data for the final slope parameters implies that the expansion dynamics in the first ultradense stage is modeled reasonably well in this approach. We thus believe that the "late" emergence of the baryon flow holds true model-independently.

One might be tempted to look for other explanations of the initial "softness" than what is provided by the RQMD model. For instance, Hung and Shuryak have put forward the idea that the system created in $\mathrm{Pb}+\mathrm{Pb}$ may be close to the so-called softest point of the EOS [30]. Initial conditions for hydrodynamical calculations may be tuned to get good agreement with the non-strange hadron spectra measured in $\mathrm{Pb}$ on $\mathrm{Pb}$ reactions [31]. However, this hydrodynamical approach fails to explain the mass dependence of the transverse flow, i.e. the data for $\mathrm{S}$ projectiles [32]. On the other side, the RQMD model provides an explanation for the systematics from $\mathrm{p}+\mathrm{A}$ and $\mathrm{S}+\mathrm{A}$ to $\mathrm{Pb}+\mathrm{Pb}[12]$. According to the RQMD model the transverse pressure during the early stages is small in all these systems, generically a pre-equilibrium signature.

In summary, we report the results of the analysis of the particle transverse momentum distributions from the central $158 \mathrm{AGeV} \mathrm{Pb}+\mathrm{Pb}$ collisions. The systematics of the baryon spectra indicates that the main component of the baryon flow develops only rather late, after most of the multi-strange particles have already frozen out. We infer from the analysis of RQMD results that characteristic energy densities at which the multi-strange hadrons freeze out are around $1 \mathrm{GeV} / \mathrm{fm}^{3}$ for central $\mathrm{Pb}$ on $\mathrm{Pb}$ collisions. Presumably, they may be formed at even larger densities.

We are grateful for many enlightening discussions with Drs. S. Panitkin, A. Sakaguchi, B. Schlei, E.V. Shuryak, and J. Sollfrank. This research used resources of the National Energy Research Scientific Computing Center. This work has been supported by the U.S. Department of Energy under Contract No. DE-AC03-76SF00098 and W-7405-ENG-36 and National Science Foundation.
[1] Quark Matter '96 proceedings, Nucl. Phys. A610, 1c (1996), and Quark Matter '97 proceedings, to appear in Nucl. Phys. A.

[2] R. Pisarski, and F. Wilczek, Phys. Rev. D29, 338(1984).

[3] L. van Hove, Z. Phys. C21, 93(1983).

[4] F. Karsch, Nucl. Phys. A590, 367c(1995).

[5] K. Kajantie, M. Laine, J. Peisa, A. Rajantie, and M. Shaposhnikov, Phys. Rev. Lett. 79, 3130(1997).

[6] H. Sorge, J. Nagle, and B.S. Kumar, Phys. Lett. B 355 , 27(1995).

[7] S. Esumi, S. Chapman, H. van Hecke, and N. Xu, Phys. Rev. C55, R2163(1997).

[8] I. G. Bearden et al., (NA44 Collaboration), Phys. Rev. Lett 78, 2080(1997).

[9] I. Kralik et al., (WA97 collaboration), talk at Quark Matter '97, Tsukuba, Japan, December 1-5, 1997.

[10] G. Roland et al., (NA49 collaboration), talk at Quark Matter '97, Tsukuba, Japan, December 1-5, 1997.

[11] D. Ferenc, Nucl. Phys. A610, 523c(1996).

[12] H. Sorge, Phys. Lett. B402, 251(1997).

[13] P. Braun-Munzinger, J. Stachel, J.P. Wessels, and N. Xu, Phys. Lett. B365, 1(1996).

[14] L.V. Bravina, I.N. Mishustin, N.S. Amelin, J.P. Bondorf, and L.P. Csernai, Phys. Lett. B354, 196(1995).

[15] H. Sorge, Phys. Lett. B373, 16(1996).

[16] A. Leonidov, M. Nardi, and H. Satz, Z. Phys. C74, 535 (1997); Jan-e Alam, J. Cleymans, K. Redlich, and H. Satz, LANL Preprint, nucl-th/9707042, (1997).

[17] U. Heinz, Nucl. Phys. A610, 264c(1996).

[18] The slope parameter is obtained from fitting the transverse mass distribution with an exponential function $\exp \left(-m_{T} / T\right)$.

[19] C. Bormann (NA49 Collaboration), J. Phys. G23, 1837(1997).

[20] P. Koch, J. Rafelski and B. Müller, Phys. Rep. 142, 167(1986).

[21] M. Prakash, M. Prakash, R. Venugopalan, and G.M. Welke, Phys. Rev. Lett. 70, 1228(1993).

[22] S. Abatzis et al., (WA94 collaboration), Phys. Lett. B400, 239(1997).

[23] Here as in the following we only refer to the transverse directions when we are discussing limits on the collective motion. Undoubtedly, nucleus-nucleus reactions at these high energies exhibit a tremendous amount of collective motion in the longitudinal direction "from the beginning" which is just the relic of the initially prepared system.

[24] F. Becattini, M. Gaździcki, and J. Sollfrank, Euro. Phys. J., in press, and hep-th/9710529.

[25] H. Sorge, Phys. Rev. C52, 3291(1995).

[26] Particle Data Group, http://pdg.lbl.gov.

[27] G.Q. Li, and C.M. Ko, J. of Phys. G22, 1673(1996).

[28] E.L. Bratkovskaya, W. Cassing, Nucl. Phys. A619, 413(1997).

[29] S.A. Bass et al., nucl-th/9803035 (1998).

[30] C.M. Hung, and E. Shuryak, Phys. Rev. Lett. 75, 4003(1995).

[31] C.M. Hung, and E. Shuryak, Phys. Rev. C57, 1891(1998).

[32] E. Shuryak, private communication, 1998. 